\title{
The Microscope as a Tool of Covid-19 Ocular Transmission Especially Between Students in Lab
}

\section{Rehab Mohamed Atta El-Desoukey*}

Associate Professor, Microbiology and Immunology Department, National Research Centre, Giza, Egypt and Biology Department, Faculty of Science and humanities in Al Quwai'iyah, Shaqraa University, Saudi Arabia

*Corresponding Author: Rehab Mohamed Atta El-Desoukey, Associate Professor, Microbiology and Immunology Department, National Research Centre, Giza, Egypt and Biology Department, Faculty of Science and humanities in Al Quwai'iyah, Shaqraa University, Saudi Arabia.

Surfaces frequently touched through human beings grow to be without problems contaminated with microorganisms. Many recent studies have addressed the microbial load and related health risks of frequently used objects, which include smart phones or money [1].

Coronavirus 2019 (COVID-19), that's as a result of severe acute breathing syndrome coronavirus 2 (SARS-CoV-2), has quickly multiplied into epidemic, elevating interest for the corona virus circle of relatives once more after the extreme acute respiration syndrome (SARS) outbreak in 2003. Although in keeping with the information obtained until now, corona virus is transmitted mainly through respiratory tracts amongst people, the position of ocular transmission was also emphasised in latest studies. Among 7 types of human corona viruses identified, three had been mentioned to be identified in ocular secretions, together with human coronavirusNL63 (HCoV-NL63), severe acute breathing syndrome corona virus (SARS-CoV) and SARS-CoV-2. Additionally, the cellular entry receptors of numerous kinds of human corona virus have been found at the ocular surface consistent with the published researches [2].

With this spread of the Corona epidemic World Health Organization and a lot of recent researches recommended taking all precautionary measures preventing the spread of disease in accordance to the previous research and among the ways of transmission of the disease through the eyes.

Microscopes are used in virtually every biological and medical laboratory. Previous cultivation-based studies have suggested that direct contact with microscope eyepieces increases the risk of eye infections [1].

More over the role that the microscope performs in transmitting bacterial and viral infections (Figure 1), in line with (McDougall., et al. 1991) who concluded that an outbreak of conjunctivitis in a microelectronics factory turned into due to transmission from infected microscopes [3]. In addition to (Olcerst, 1987) who indicates that direct contact with microscope eyepieces presents a probably significant path of transmission of both bacterial and viral ocular infections. However, sharing optical devices may be greater problematic. Previous, cultivation-primarily based research. Suggested that direct contact with microscope eye-pieces significantly increases the risk of reoccurring eye infections, such as conjunctivitis. $26 \%$ of the investigated oculars carried bacteria known to be pathogenic or doubtlessly pathogenic, which includes Staphylococcus aureus [4].

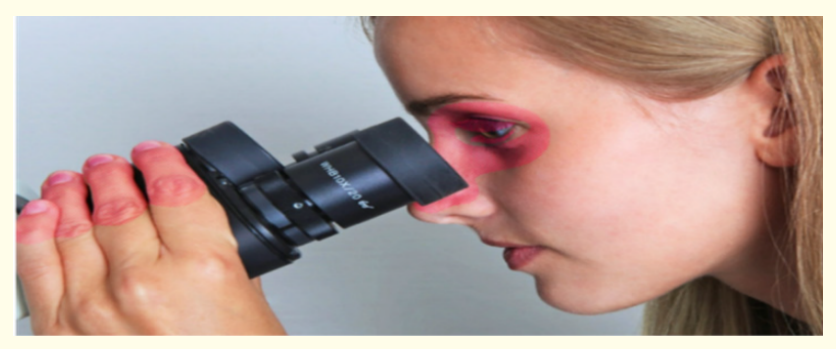

Figure 1: Skin and eye areas (highlighted in red) with probable contact to microscope oculars. Photographs with permission of Furtwangen University [1]. 
While the possibility of transmission of the virus through the eye has been documented in a small percentage within the research, it is the subject of future studies to reinforce the matter or not, but the necessary precautions and measures should be taken in light of the spread of an epidemic, and all methods leading to its spread must be excluded, even if they are possible and from the above mentioned studies it is clear that the microscope oculars definitely have the potential to act as carriers of infectious pathogens.

As many countries have made distance education as a preventive measure for the spread of Covid 19 epidemic, but with the advent of vaccines, some countries began to return to the study again, especially for students of scientific and medical specializations because they need laboratories and practical application to achieve the highest educational outcomes but this matter without taking precaution make the microscope play a problematic source of infection especially during practical examinations as microscope ocular lens used by students, whether using a lens sterilizer in-between or without using a sterilizer, as it sometimes require students to pass in succession to microscopes to demonstrate the slides without any sterilization between the student and the next.

The good news: cleaning and sterilization reduces the number of germs significantly by $99 \%$. This should be carried out regularly, particularly if different people use the same microscope. This is according to a current paper which compared distinct healthcare germicides. found that people with $70 \%$ concentration ethanol had a stronger impact on specific corona viruses (mouse hepatitis virus and transmissible gastroenteritis virus) after one minute touch time on difficult surfaces while as compared with $0.06 \%$ sodium hypochlorite. Tests done the usage of SARS-CoV showed that sodium hypochlorite is powerful at a concentration of 0.05 and zero. $1 \%$ after five minutes while it's far blended to an answer containing SARS-CoV. Similar effects had been obtained the usage of household detergents containing sodium lauryl ether sulphate, alkyl polyglycosides and coco-fatty acid diethanolamide [5].

It is also worth noting that sterilization of microscope lenses, which can reduce the microbial content of ophthalmic lenses, but according to many studies has side effects on eye health as mentioned by (Olcerst, 1987) Sterilization of eyepieces by ethylene oxide, formaldehyde and isopropyl alcohol were considered, but ultimately rejected. These biocides were found respectively to damage ocular lens coatings, contribute to volatile organic emissions, or be ineffective against spore-forming bacteria. This article also presents a detailed assessment of a commercially to be had ultraviolet sanitization unit (synthetic through the King Bactostat Corp., 7115 Armistad Street, El Paso, TX 79912). This ultraviolet disinfection technique proved to be fast and emission unfastened; it additionally yielded eyepieces freed from residual chemical biocides which have the ability for ocular infection. Field assessments involving 60 eyepieces tested effective disinfection by way of a Chi-Square statistical contrast, at values greater than 95\% self assurance level, in comparison to unirradiated eyepieces [4].

From all the above it can be concluded that the possibility of transmission of eye infection through the eyepiece of the microscope, whether bacterial or fungal, in addition to the Covid 19.

Recommendations for the occupational health, researchers and students taking all precautionary measures while using microscopes in hospitals, research centers, laboratories of medical and scientific departments in universities and science laboratories in schools using safety glasses, gloves and periodical sterilization either by sterilizer or ultraviolet sanitation (see Figure 2).

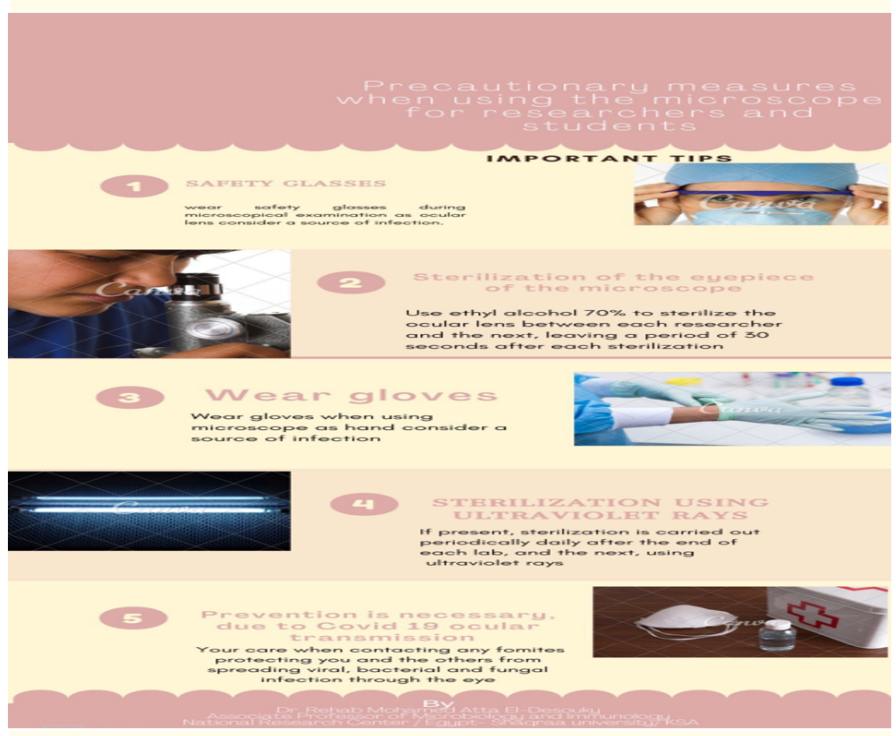

Figure 2: Precautionary measures when using the microscope for researchers and students.

In addition, increasing the awareness by initial and periodic educational presentations to students, researchers and lab work- 
ers and management by the occupational health are essential to reinforce the potential health problem that exists and recommendations for prevention.

\section{Bibliography}

1. Fritz B., et al. "Eye-catching microbes-polyphasic analysis of the microbiota on microscope oculars verifies their role as fomites". Journal of Clinical Medicine 9.5 (2020).

2. Shen J., et al. "The paradoxical problem with COVID-19 ocular infection: Moderate clinical manifestation and potential infection risk". Computational and Structural Biotechnology Journal 19 (2021).

3. McDougall C., et al. "Conjunctivitis in clean room workers: Nursing evaluation and intervention". AAOHN Journal 39.1 (1991).

4. Olcerst RB. "Microscopes and Ocular Infections". American Industrial Hygiene Association Journal 48.5 (1987).

5. Lai MYY., et al. "Survival of severe acute respiratory syndrome coronavirus". Clinical Infectious Diseases 41.7 (2005): e67-e71.

Volume 4 Issue 12 December 2021

(C) All rights are reserved by Rehab Mohamed Atta

El-Desoukey.

Citation: Rehab Mohamed Atta El-Desoukey. "The Microscope as a Tool of Covid-19 Ocular Transmission Especially Between Students in Lab". Acta Scientific Microbiology 4.12 (2021): 101-103. 